\title{
Research on Scheduling Algorithm Based on Elevator Group Control System
}

\author{
XueXia Zhang ${ }^{1, a}$ An Ying Fu ${ }^{1, b}$ and JinBo Zhang ${ }^{1, c}$ \\ ${ }^{1}$ Shaanxi institute of intenational trade\&commerce.xian,China \\ a187383930@qq.com, 'b690061598@qq.com, c526165466@qq.com
}

\begin{abstract}
Keywords:Elevator group control system Multi - objective optimization algorithm Delayed Flight Algorithm
\end{abstract}

Abstract. This paper is based on the elevator group control system scheduling algorithm research, For the current elevator running in the process of a long time ladder phenomenon,analyzes the structural characteristics of the group controlled elevator system, puts forward the structural scheme of the overall design of the elevator group control system, realizes the communication between the elevator group controller and the single one, and carries on the unified coordination dispatching to the single elevator. Taking into account the elevator ladder in the process of waiting time, ladder time, energy loss and other issues, as well as different modes of traffic operation, etc., proposed to adapt to the situation of the most tactics, multi-objective optimization algorithm and delay And it is applied to the scheduling strategy of elevator group control. The scheduling algorithm is implemented by software program.

\section{Introduction}

At present, in a variety of shopping malls and buildings, elevators have become an indispensable important construction equipment,for improving the quality of life of the people, in the realization of energy-saving provincial policy has played an irreplaceable support role ${ }^{[1]}$. With the development of society, large buildings, especially large-scale office buildings, Only a single elevator is not very good to deal with all the passenger flow, so need to set up several or more elevators, and ensure that each elevator control system interlocking. Also take into account the peak hours of commuting, traffic is relatively large, relying solely on the increase in elevator load, speed, the number of stations, it is difficult to overcome the frequent operation of the elevator car, but also can not improve in a certain period of time must appear long time ladder phenomenon. This requires solving the problem of coordinated scheduling of the elevator group.

\section{Elevator group control system}

\section{Characteristics of elevator group control system}

Elevator group control system refers to an elevator group that is compositing of a number of independent operation of the elevator that according to the actual function of the building and the population density, by the computer control system for the elevator group unified management and distribution, And according to the system set to run the traffic state algorithm, to achieve the most escalator elevator program.

At present, there are two kinds of elevator group control system, one is the elevator full control system that has been configured in advance, which is embedded inside the elevator system, the data stored in memory. The other is in the external implementation of the elevator, Through the 
interception of the call outside the elevator,and then unified distribution of the signal to achieve the elevator group control system.

In the elevator group control system, when a layer of call information appears, the signal is not transmitted directly to the central host, but through the group control system host algorithm analysis, to find the optimal response, That is, to select the most suitable for the call of the elevator from the elevator group, and send the order to the elevator host, to execute this response. Through the actual traffic conditions, the elevator to a reasonable scheduling, so as to improve the efficiency of the elevator to save waiting time. However, in order to ensure the stable operation of the group control system, the elevator group control system only to control the external signal, for the call signal ,only to collect without control .

In addition, when the elevator is grouped, it is necessary to take into account the failure of a certain elevator or group control host, so in design of the group control system, to consider the situation that the elevator is not subject to group control. In the normal operation of the elevator, the ladder controller needs to determine whether the elevator to accept the group control, if so, through the CAN bus pass the data to the group control host, by the group control host to unified scheduling, assign the best elevator to respond to this call signal; Otherwise, the single-ladder controller responds directly to this signal. When an elevator is in a fault condition, the elevator will be prohibited from group control ${ }^{[2-4]}$.

\section{Design of Group Control System}

In the group control system, should keep the added group control system in the control logic independence, and with the original elevator system there is electrical signal isolation, to avoid mutual interference. The system does not make any treatment inside the elevator, only in the original elevator system, Install the equipment to intercept the call signal, and the communication equipment, realize the two-way communication between the elevator and the group control host.

By adding a control system in the original elevator system to achieve group control, considering the different elevator model, you can use the interface layer to receive the signal of different elevators to be encoded and sent in the same information format, then the group will receive the same format of the signal, so as to achieve the different elevators control.

Elevator group control system can be divided into three parts, group control system, communication system and signal acquisition system, the system block diagram shown in fig 1 .

Elevator group control host is the core of the entire control system, which is mainly responsible for the coordination and control of the entire system and optimize the scheduling, through a single ladder system to complete a variety of elevator signal acquisition, sent to the main control system, to analyze and generate scheduling commands, then sent to the corresponding elevator control system response. When a new station call request is generated, according to the group control algorithm, the group control host achieve the optimal escalator, after the new station call response, the response to the past floor not have much impact, otherwise, will affect comfort of waiting passengers. A command system for monitoring host and group control systems together to achieve the elevator group in the elevator system status monitoring work, easy to detect system hidden trouble, troubleshooting, at the same time, which can configure elevator parameters to facilitate the backstage management of elevator. ${ }^{[5]}$

The communication of the group control system mainly refers to the communication between the control board and the group control host, and the system realizes the data communication through the CAN bus. 


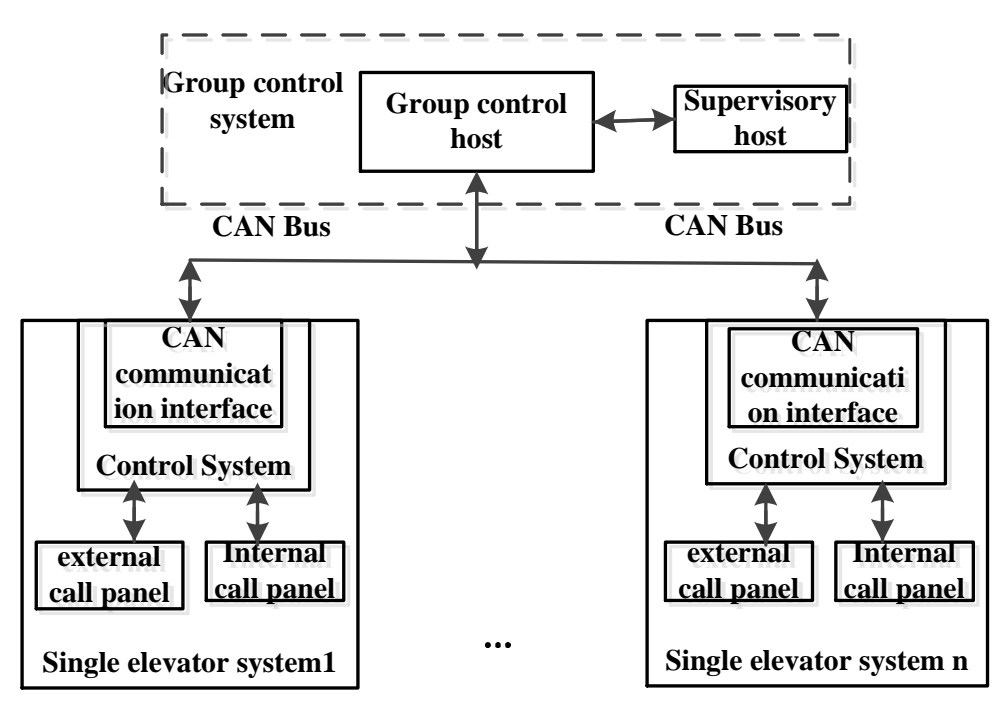

Fig1 Elevator block control system structure block diagram

In addition, which also includes the communication between the group control host, the call control board and the state control board, however, communication between host and an outer call plate of a single elevator is less.

The signal acquisition is the basis for elevator group control, a single elevator (ie elevator control system) is the basic unit of the entire data acquisition system, each elevator real-time collects information of call and summon, and send these data to the outside call control panel, which directly communicate with the group control host, then the host achieve the corresponding processing. Signal acquisition and control circuit structure diagram shown in fig2.

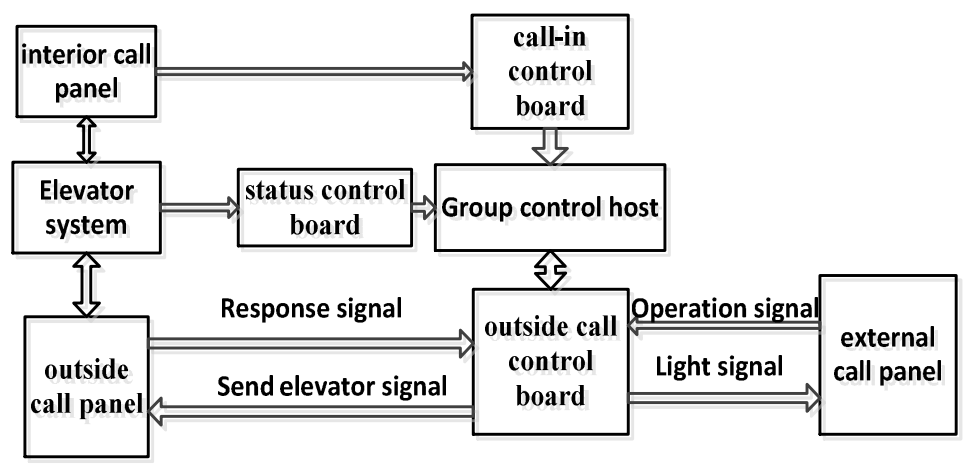

Fig2 Signal acquisition control circuit structure diagram

The outside call panel and the outside call control board is based on the original elevator system to increase, the external control panel is data processing board that controlled by the independent CPU, the outside call board will be signal acquisition, after the result of the host processing that be send by the outside call control panel, the external call board button signal really play a role. When the group control system fails, the external control panel only plays the role of signal transmission, no longer accept the group control host scheduling signal. When the group control system fails, the external control panel only plays the role of signal transmission, no longer accept the group control host scheduling signal.

The call-in control board and the status control board are responsible for collecting the incoming call signal and real-time running status information of the elevator, after receiving the command from the group control host, send the elevator signal to the bus, they only capture the signal does not receive the host control. 


\section{Multi - objective Optimization Group Control Scheduling}

For the elevator group control system, often composed of multiple elevators, is a multi-objective task system. Single elevator system to independently complete the call, the signal of outside call is unified calculated by the group control system, from the calculated best single elevator to respond to this call signal. For the group control system, in the process of achieving group control to achieve multi-objective optimization, so as to solve the elevator multi-objective optimization group control scheduling problem.

\section{Evaluation function}

Multi-objective optimization adopts the method of target combination, and combines the multi-objective into a new target in different ways, and then uses the single-objective optimization method to solve. In the multi-objective optimization method, this paper adopts the linear weighting in the simplest evaluation function to realize multi-objective optimization.

First, according to the importance of the target $\mathrm{p}_{\mathrm{r}}$, give a set of weights $\left(\lambda_{1}, \lambda_{2}, \lambda_{3}, \ldots, \lambda_{\mathrm{n}}\right)^{\mathrm{T}}, \lambda_{\mathrm{t}} \geq 0, \mathrm{t}=1,2, \ldots, \mathrm{n}, \sum_{\mathrm{t}=1}^{\mathrm{n}} \lambda_{\mathrm{t}}=1$, each objective function is weighted into a new objective function, that is, the evaluation function is

$$
F(x)=\min \sum_{t=1}^{n} \lambda_{t} p_{t}(X)
$$

Where $p_{8}(X)$ is the combined objective function. Different combinations of method construct a combined objective function,, the elevator i response to the j-layer call signal evaluation function can be expressed as

$$
F(i, j)=\lambda_{1} p_{1}(i, j)+\lambda_{2} p_{2}(i, j)+\lambda_{n} p_{n}(i, j)
$$

Where $p_{t}\left(i_{i}\right)$ is an optimal evaluation function estimated by the elevator $\mathrm{i}$ in response to the $\mathrm{j}$ layer call signal, the smaller the value, the better the group control scheduling method of the elevator. $\lambda_{\mathrm{r}}$ is a importance weight coefficient, by changing the value of each $\lambda_{\mathrm{r}}$, adapt to different elevators of different traffic passenger flow mode, so that group control scheduling to optimize.

\section{Multi - objective Optimization Group Control Scheduling Algorithm}

In the normal operation time of the elevator, the elevator traffic mode can be divided into normal traffic mode, idle traffic mode and peak traffic mode, The scheduling algorithm of normal traffic mode mainly considers the short time of waiting time, taking proper consideration of the time of flight and the energy loss of the system. According to the multi-objective optimization algorithm, the optimal response ladder is obtained. According to the rules of the elevator to get expert experience, setting a different weighting factor can change the elevator group control scheduling algorithm $^{[6-7]}$. Multi-objective optimization group control scheduling algorithm shown in figure 3 . Where $\mathrm{M}$ is the number of single steps involved in group control, Tr represents the time required for the elevator to run, Ts represents the average dwell time of the elevator on each floor, and $n$ 
represents the target floor to which the elevator is to arrive.

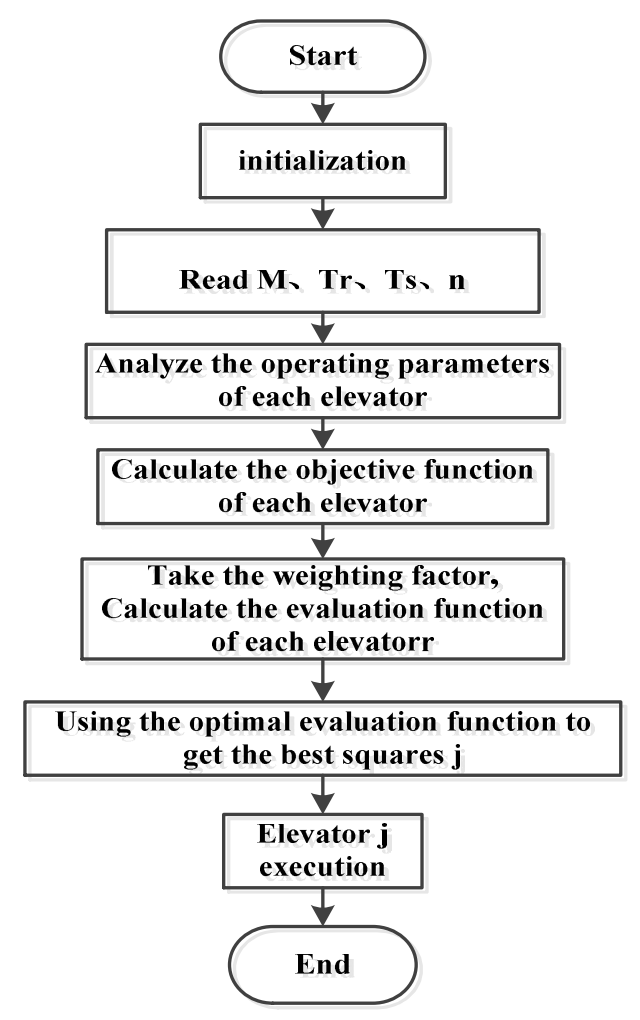

Fig3 Multi - objective Optimization Group Control Scheduling Algorithm

In the idle period, only a very few floors have fewer passengers need to service, this time only a small number of elevators put into use, play the purpose of saving energy. Turn off some of the elevator, in the external call signal for attribute analysis, and then according to the algorithm in 3 to carry out the deployment of the elevator, the algorithm only need to change the weighting factor. ${ }^{[8]}$

\section{Delayed ladder selection figure algorithm}

Before proceeding with the selection, the group control system needs to calculate the waiting time of the new incoming signal response, taking into account the time delay caused by the response of the signal to other external signals. The external call signal and the status of the elevator is also real-time changes, based on this consideration, in order to minimize the average waiting time for passengers, so delay selection algorithm is proposed ${ }^{[9,10]}$. In this algorithm, after sent the external call signal of a certain floor of sent, the call outside control panel to intercept the request signal, by the master system for repeated calculation to choose the best elevator, at the appropriate time, the main control system will send this station signal immediately sent to the optimal elevator to respond, the delay algorithm is shown in figure 4. The incoming call signal is detected from the first layer, and when the service signal of the elevator is left alone, the escort request is sent to the group master. 


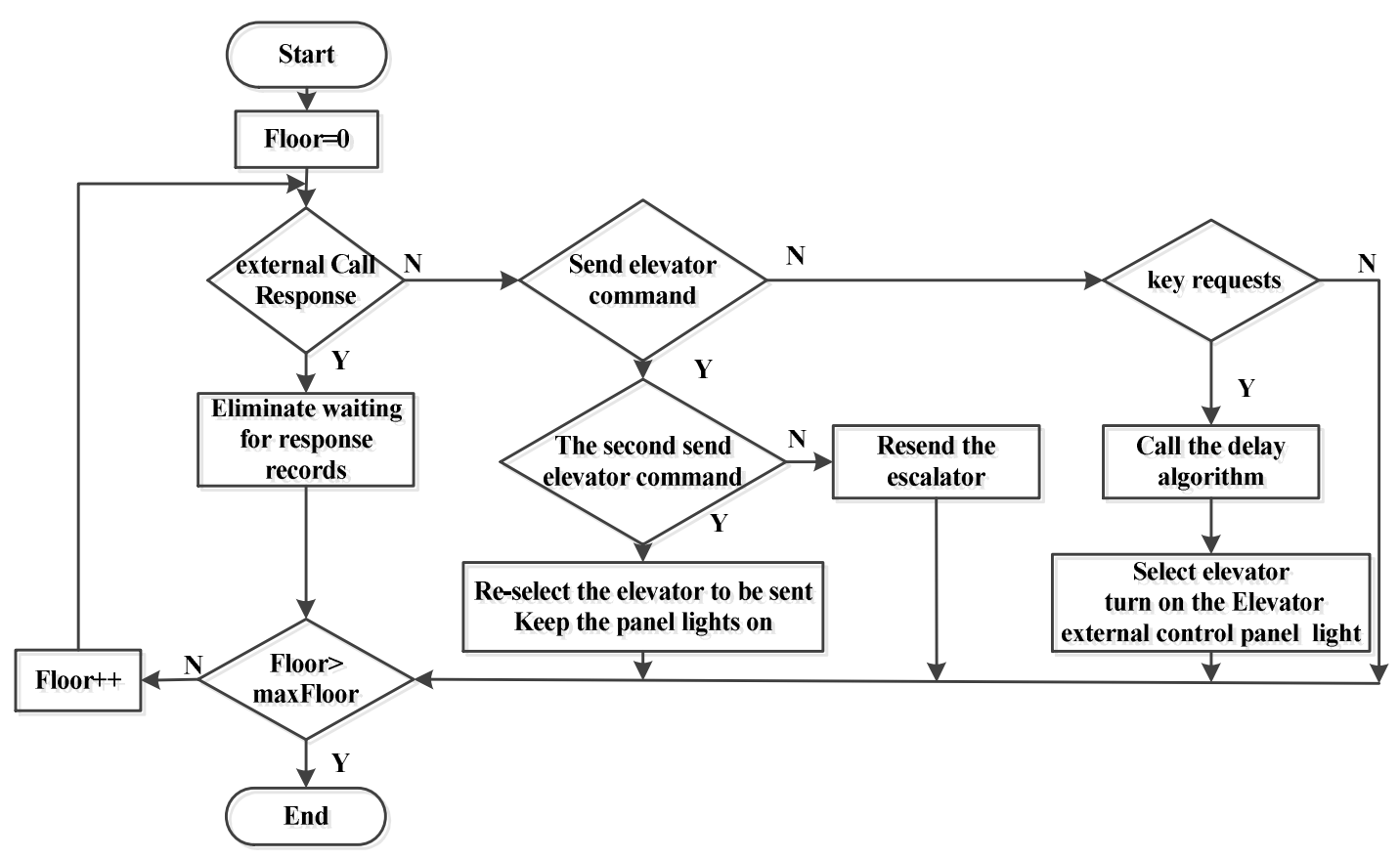

Fig 4 Delayed Flight Algorithm

\section{Group control scheduling algorithm}

Group control scheduling strategy in the course of work, the external call signal sent to the group control host, through the group control system software to send data to the scheduling rules module, the group control data from the initial processing, It is determined whether the external call signal enters the group control data. If so, it is selected by the scheduling rule according to the traffic mode set by the system, so as to select what kind of school algorithm to adopt, and then call the corresponding algorithm to calculate the optimal allocation elevator. According to the elevator expert knowledge or experience, you can not calculate the results directly. This can reduce the amount of system operations, improve the efficiency of group control system ${ }^{[11,12]}$.

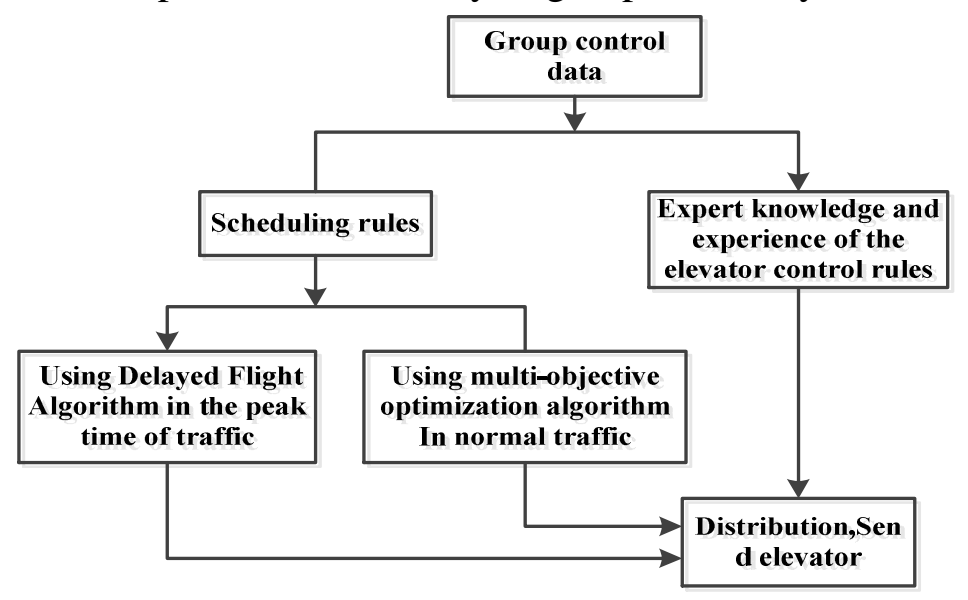

Fig5 Group control scheduling strategy

Group scheduling strategy as shown in figure 5, the scheduling rules for the call to the algorithm to take the peak traffic, traffic is relatively large time, use delayed flight algorithm to ensure that the passenger waiting time is the shortest, other traffic hours, adopt target optimization algorithm, equalize the elevator running parameters, such as: elevator energy loss. 


\section{Summary}

Through the analysis and design of the overall structure of the elevator group control system, the paper puts forward the centralized control and coordination of the single ladder, improves the utilization rate of the elevator and ensures the comfort of the passengers. At the same time, two kinds of optimal algorithms are proposed in the coordinated control of the elevator group, and it is applied to the scheduling strategy of the group control system. Through the group control software program and data communication, the group controller is used to obtain the running state parameters of each ladder, and the single ladder is unified.

In order to meet the requirements of the group control system, the group control system software program can realize the centralized and reasonable scheduling of the elevator group, and can monitor the running status of the elevator group and display the running status of each elevator ,to detect the failure of the elevator, and in time to exclude it outside the group control system.

\section{Acknowledgements}

This work was financially supported by Shaanxi Provincial Department of Education Research Project (15JK2020).

\section{Reference}

[1] Tao.Li: The Design and Realization of Elevator Control System Based on PLC, edtied by EIC(2010).

[2] TianMing.Liu: The Intelligent Elevator control system, edtied by Electrical and Mechanical Engineering(2004).

[3] Chao.Liu and XiaoPin.Ma: The Design of Elevator Automatic Dispatching Control System Based on PLC, edtied by Technology Development of Enterprice(2017).

[4] Jin.Ou: The Research and Design of Elevator Control System Based on CAN Bus, Low Voltage Appartcs (2012).

[5] Yun.Shi: The Design and Implementation of Elevator Control System Based on PLC, edtied by Industrial Control Computer(2009).

[6] DaLong.Ma: The Design and Research of Elevator Group Control System Based on Ant Algorithm, Jilin University(2013) .

[7] ZenChan.Li: The Research on Elevator Intelligent and Group Control Strategy, Tianjin University(2014) .

[8] GuanNan.Li: The Circuit Design of Multi-storey Elevator Control System, New Technology \& New Products of China(2010)

[9] Zhe.Ou: The Research and Design of Intelligent Elevator Control System Based on Electrical and Mechanical Engineering Technology, Journal of Mechanical and Electrical Engineering(2006). [10] Lin.Lin: The Research on Multi - objective Intelligent Optimal Scheduling Algorithm for Group - controlled Elevator, Northeastern University(2005) .

[11] JingWei.Sun: The Research of Elevator Control System Based on PLC and Fuzzy Control, Manufacturing Industry Automation(2011).

[12] YanHua.Li: The Research and Design on Elevator Intelligent and Group Control System, Xiamen University(2008). 